\title{
Commissioning of the ATLAS level1 endcap muon trigger system
}

\author{
S.X. Oda, on behalf of the ATLAS Collaboration
}

The University of Tokyo, International Center for Elementary Particle Physics, 7-3-1 Hongo, Bunkyo-ku, Tokyo 113-0033, Japan

\begin{abstract}
The ATLAS level1 endcap muon trigger system at the LHC selects interesting events containing muons with transverse momenta greater than $6 \mathrm{GeV} / c$ from $40 \mathrm{MHz}$ proton-proton collisions. This system consists of 3588 Thin Gap Chambers (TGC) and the total number of readout channels is $3.18 \times 10^{5}$. The trigger logic is based on the coincidence between 7 layers of TGC. All processes are performed using hardware within the maximum latency of $2.5 \mu$ s for the level1 trigger. To be ready for the first beam collisions scheduled in 2009, we have succeeded in sending trigger signals of cosmic ray muons with the synchronous operation at $40 \mathrm{MHz}$ and fine signal timing alignments. During the single proton beam circulation at the LHC in 2008, we have also confirmed that the timing of the endcap muon trigger for tracks of beam halo muons produced at the collimator was well aligned. We report on status of the commissioning and results from runs with cosmic ray muons and single beams.
\end{abstract}

Key words:

Trigger, Muon, Thin gap chamber, The ATLAS experiment

\section{ATLAS level1 endcap muon trigger system}

\subsection{ATLAS experiment}

The ATLAS experiment is one of the big experiments at the Large Hadron Collider (LHC) at CERN to find and study Higgs boson and new physics [1]. Proton beams will be collided at $\sqrt{s}=14 \mathrm{TeV}$ with $25 \mathrm{~ns}$ bunch crossing intervals. One bunch contains $10^{11}$ protons and instantaneous luminosity will be $10^{34} \mathrm{~cm}^{-2} \mathrm{~s}^{-1}$. With this instantaneous luminosity, more than 20 collisions occur per bunch crossing and integrated luminosity will be $100 \mathrm{fb}^{-1}$ per year.

\subsection{Muon system}

Since major decay branches of the standard model Higgs boson except $H \rightarrow \gamma \gamma$ have at least one muon in the final N state, muons are important at the ATLAS experiment [2]. Under the harsh environment, the following specifications are required for the ATLAS muon system:

- Radiation-hard electronics and sensor elements having fast response

- Large acceptance in pseudorapidity $(\eta)$ with the full azimuthal $(\phi)$ coverage

- Good muon identification and transverse momentum $\left(p_{T}\right)$ resolution over a wide range of $p_{T}$

$$
-\sigma_{p_{T}} / p_{T}=10 \% \text { at } p_{T}=1 \mathrm{TeV}
$$

- Highly efficient triggering on low $p_{T}$ objects with sufficient background rejection
The ATLAS muon system consists of four kinds of muon chambers and toroidal magnets. Barrel and endcap toroidal magnets bend muons in the $\eta$ direction for the momentum measurements. Monitored Drift Tube (MDT) performs precision tracking in the $\eta$ coordinate in the barrel and endcap region $(|\eta|<2.7,|\eta|<2.0$ for the innermost station). Cathode Strip Chamber (CSC) provides 2-dimensional tracking $(\eta$ and $\phi)$ in the forward region $(2.0<|\eta|<2.7)$ in the innermost station. Thin Gap Chamber (TGC) and Resistive Plate Chamber (RPC) provide triggers and the second coordinate $(\phi)$ in the endcap region (1.05<| $<|<2.7,| \eta \mid<2.4$ for triggering) and the barrel region $(|\eta|<1.05)$, respectively. The numbers of chambers and readout channels of TGC are 3588 and $3.18 \times 10^{5}$, respectively.

\subsection{Thin gap chamber}

TGC is a wire chamber and works in the limited proportional mode. The anode-to-cathode distance of $1.4 \mathrm{~mm}$ is smaller than the distance between anode wires of $1.8 \mathrm{~mm}$. Signals are read out by anode wires and cathode strips in the $\eta$ and $\phi$ directions, respectively. Gas mixture of $\mathrm{CO}_{2} / \mathrm{n}$-pentane $(55 / 45)$ is circulated in the chamber and high voltage (HV) of $2900 \mathrm{~V}$ is nominally applied to anode wires. TGC has the following advantages and satisfies the above requirements.

- Fast response: $<25 \mathrm{~ns}$

- High efficiency: > $98 \%$

- Radiation hardness: $\sim 0.6 \mathrm{C} / \mathrm{cm}$ 
- High rate capability: $>1 \mathrm{kHz} / \mathrm{cm}^{2}$

There are 2 (TGCI), 3 (TGC1), 2 (TGC2) and 2 (TGC3) layers in four stations from the interaction point (IP). The last three stations are called the TGC big wheel and used for triggering.

\subsection{Trigger system}

The ATLAS trigger system selects interesting events from a large amount of proton-proton collision events with three steps, level1 (L1, hardware), level2 (software) and event filter (software). The level1 identifies the signatures of muon, electron/photon, tau, jet and missing $E_{T}$ with adjustable threshold sets. The level1 works as a pipeline trigger system with the $40 \mathrm{MHz}$ clock, synchronous to the LHC proton-proton collisions. The processing latency is required to be less than $2.5 \mu \mathrm{s}$. The level1 rate is reduced to $75 \mathrm{kHz}$ from $40 \mathrm{MHz}$. The level1 trigger system consists of three sub systems, L1 muons, L1 calorimeters and Central Trigger Processor (CTP). L1 muons consists of the endcap muon trigger system with TGC, the barrel muon trigger system with RPC and Muon-CTP Interface (MUCTPI). MUCTPI combines information of muon triggers from endcap and barrel, and sends it to CTP. CTP generates level1 trigger decision (L1A) with the trigger information from MUCTPI and L1 calorimeters, and delivers L1A to all the ATLAS sub detectors.

\subsection{Level1 endcap muon trigger system}

The level1 endcap muon trigger system selects high $p_{T}$ muons with three steps with the bending power of toroidal magnets. At the first step, 2 (1)-station coincidence of TGC2 and 3 (TGC1) for low $p_{T}$ muons is required in the SLave Board (SLB). Coincidences of in the $r$ (the radial coordinate) and $\phi$ directions are independently required. At the second step, 3-station coincidence in $r$ or $\phi$ for high $p_{T}$ muons is required in the High PT board (HPT). At the third step, $r-\phi$ coincidence is required in the Sector Logic (SL). SL uses coincidence windows of sagitta $\delta r$ and $\delta \phi$, defined with IP, TGC1 and TGC3, to determine $p_{T}$. Adjustable six $p_{T}$ threshold levels can be used and the nominal values are $p_{T}=6,8,10,15,20$ and $40 \mathrm{GeV} / c$. The expected rate at the luminosity of $10^{33}\left(10^{34}\right) \mathrm{cm}^{-2} \mathrm{~s}^{-1}$ with the $6(20) \mathrm{GeV} / c$ threshold is $17.3(12.9) \mathrm{kHz}$ [2].

\subsection{Endcap muon trigger electronics}

Figure 1 shows a schematic view of the electronics for the endcap muon trigger system. The electronics are separately located on the detectors, at the edges of the big wheels and in the electronics hut. Twisted pair cables, $\sim 10 \mathrm{~m}$ in length, are connected between the detectors and HSC crates on the wheel edges. Optical fibers, $\sim 100 \mathrm{~m}$ in length, are connected between the HSC crates and the electronics hut. There are five types of lines for the electronics, trigger, readout, control, TTC (Timing Trigger

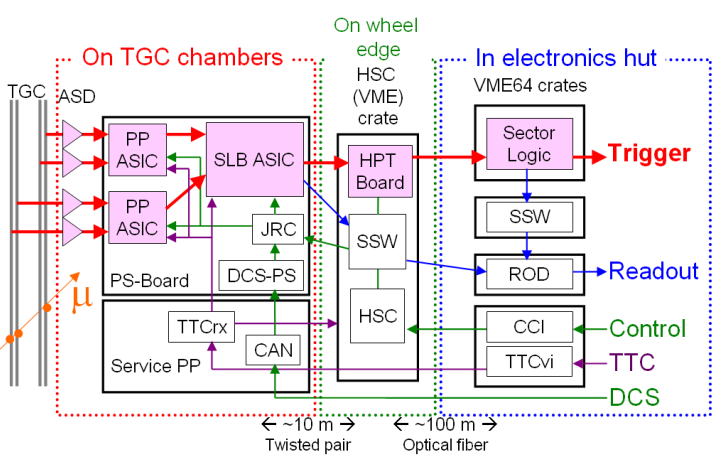

Figure 1: A schematic view of electronics of the endcap muon trigger system.

and Control) and DCS (Detector Control System). Amplifier Shape Discriminator (ASD) is mounted on the detector. The number of dead readout channels is 65 and corresponds to the fraction of $\sim 2 \times 10^{-4}$. PS (Patch Panel (PP) and SLB) pack is on the big wheel. PP has ASIC to compensate differences of the time of flight and the cable length with sub-ns step delays, and to provide test pulses for ASD. SLB has ASIC to require 2 or 1-station coincidence for triggering and to have the pipeline depth of 128 for readout. HPT requires 3 -station coincidence with small sagitta $\delta r$ or $\delta \phi$. Star SWitch board (SSW) compresses and encodes data, and sends it via optical fibers to Read Out Drivers (ROD) in the electronics hut. In the HSC crate on the wheel edge, HPT and SSW are located. SL boards are located in the electronics hut and send trigger information to MUCTPI. One ROD receives data from 8 SSW boards. It checks data consistency and converts data from the TGC specific format to the ATLAS common format. Data is sent to Read Out System (ROS) via optical fibers. Sampled events are also sent to online monitoring PCs via Ethernet cables.

\section{Commissioning of endcap muon trigger system}

\subsection{Timing alignment with test pulses}

Alignment of signal timing for all the $3.18 \times 10^{5}$ channels within one bunch crossing ( $25 \mathrm{~ns}$ ) is required for triggering. There are six kinds of variable delays for the timing alignment. The intrinsic time jitter of TGC is about $25 \mathrm{~ns}$ at the maximum and depends on the incident angle and chamber type. We need to use appropriate gate widths between 25 and $50 \mathrm{~ns}$. The difference of the time of flight can be calculated from geometry and the difference of the signal cable length between ASD and PP can be measured with test pulses. These differences are 50 ns at the maximum and are absorbed by PP ASIC signal delays with $0.84 \mathrm{~ns}$ steps. The difference of propagation of signal cables between SLB and HPT is absorbed by delays at HPT receivers with $1 / 2$ clock units (edge selection). The difference of the fiber length of TTC signals between TGC chambers and the electronics hut is about $200 \mathrm{~ns}$ and 
is absorbed by TTCrx delays with 100 ps fine steps and 25 ns coarse steps. The difference of arrival timing from SL boards is corrected at MUCTPI. The timing alignment between channels in various layers for beam collisions has been conducted with test pulses. Trigger and readout lines have been checked with about 3000 track patters generated by test pulses.

\subsection{Commissioning with cosmic ray muons}

From August to October in 2008, ATLAS continuously took cosmic ray muons with all sub detectors. More than $99 \%$ TGC chambers could be operated at $\mathrm{HV}$ of 2800 V. Problematic chambers are being replaced during the 2008/2009 winter shutdown. The endcap muon trigger system provided three kinds of triggers, one 2-station coincidence trigger (TGC_HALO) and two 3-station coincidence triggers (TGC_MU0 with large $|\delta r|$ and TGC_MU6 with small $|\delta r|)$ to other sub detectors. The typical rates are $16 \mathrm{~Hz}, 2 \mathrm{~Hz}$ and $2 \mathrm{~Hz}$ for TGC_HALO, TGC_MU0 and TGC_MU6, respectively, and were very stable. Data was continuously monitored with data quality monitors. The monitors show hit profiles (see Fig. 2), timings (hits and triggers) and efficiency of TGC, and correlations between TGC and MDT. Efficiency per layer including the loss by support materials was measured as a function of $\mathrm{HV}$ divided by the atmospheric pressure. As shown in Fig. 3, the efficiency seems to reach the plateau at $2800 \mathrm{~V}$. The nominal (design) $\mathrm{HV}$ value is $2900 \mathrm{~V}$ and the optimal operation point is under investigation.

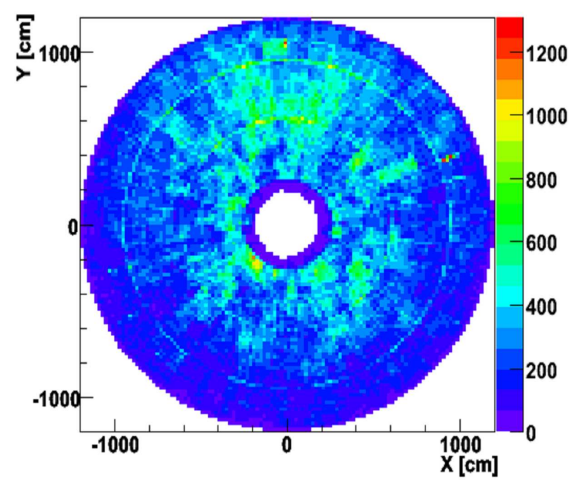

Figure 2: TGC hit profile for the $\mathrm{C}$ side $(\eta<0)$. Hit positions are calculated from coincidence of anode wire hits and cathode strip hits.

\subsection{Commissioning with single beams}

We had single $450 \mathrm{GeV}$ proton beams for 3 days from the 10 th of September, 2008. Figure 4 shows the timing of the three kinds of endcap muon triggers against the beam trigger of the counter clockwise beams (BPTX2) without $\mathrm{RF}$ capture. The timing was well aligned internally and the time of flight between two endcaps $(30 \mathrm{~m}=100 \mathrm{~ns}=4$ bunch crossings) was seen. The total rate of endcap muon triggers was about $20 \mathrm{~Hz}$. After RF was turned on, the rate became less than $0.01 \mathrm{~Hz}$. We need beams to adjust

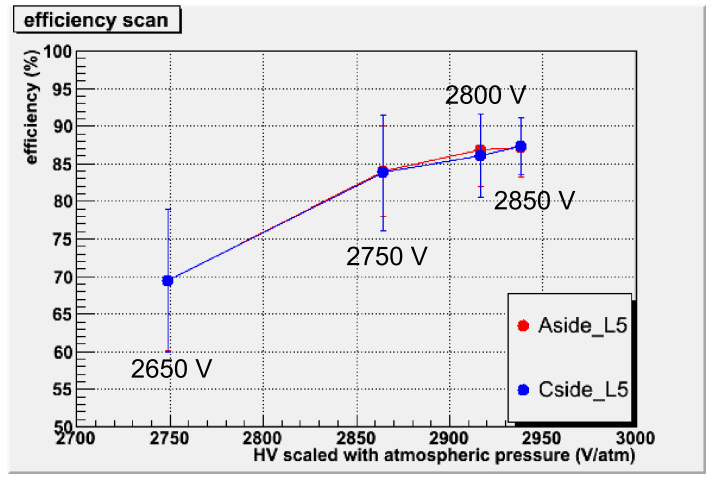

Figure 3: Efficiency of anode wires in the second layer of TGC2 of A $(\eta>0)$ and $\mathrm{C}(\eta<0)$ sides. Bars on data points represent the RMS of efficiency values.

the timing of endcap muon triggers against beams. It is just single parameter adjustment.

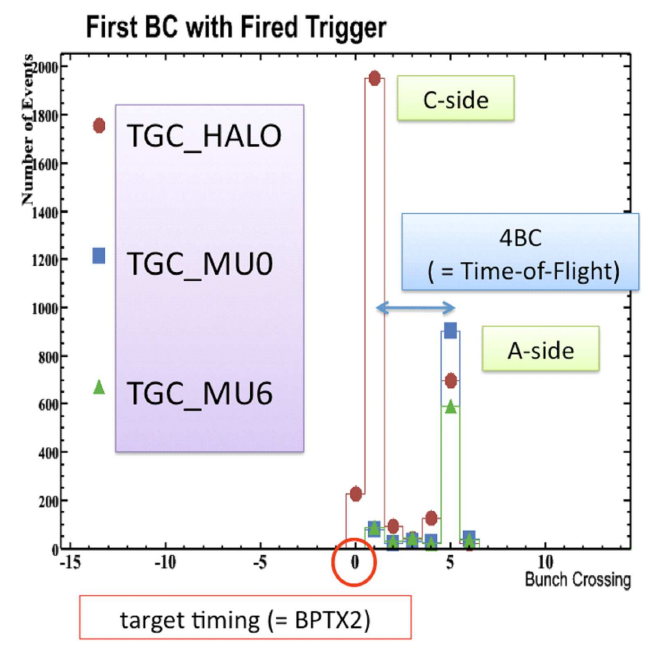

Figure 4: The timing of three kinds of endcap muon triggers against the beam trigger of the counter clockwise beams (BPTX). The timing was well aligned and the time of flight between two endcaps was correctly seen.

\section{Summary}

The level1 endcap muon trigger system of the ATLAS experiment with final infrastructures has been commissioned with test pulses, cosmic ray muons and single beams. More than $99 \%$ TGC chambers are working. The timing of $3.18 \times 10^{5}$ channels has been well aligned internally. The system is ready for $p p$ collisions at $\sqrt{s}=10 \mathrm{TeV}$ which will be started from autumn in 2009 .

\section{References}

[1] The ATLAS Collaboration, G. Aad et al., JINST 3 (2008) S08003.

[2] The ATLAS Collaboration, G. Aad et al., arXiv:0901.0512 [hepex]. 